Pacific Journal of Mathematics

TENSOR AND DIRECT PRODUCTS 


\section{TENSOR AND DIRECT PRODUCTS}

\section{CARY WeBB}

Let $R$ be an associative ring with $1, E$ a unitary right module, and $\left(F_{i}\right)_{i \in I}$ a family of unitary left modules. Let $f: E \otimes_{R} \Pi F_{i} \rightarrow \Pi\left(E \otimes_{R} F_{i}\right)$ be the canonical map. Theorem. $f$ is bijective (surjective) for all families $\left(F_{i}\right)$ iff $E$ is finitely presented (finitely generated). Theorem. If $R$ is a Dedekind domain or is commutative artinian and every $F_{i}$ is flat, then $f$ is injective. Corollary. If $R$ is a Dedekind domain or is commutative artinian, every $F_{i}$ is flat and $E \bigotimes_{R} F_{i}$ is reduced, then $E \otimes_{R} \Pi F_{i}$ is reduced. Theorem. If $R$ is a Dedekind domain or is commutative artinian, $E_{j}$ is flat, $f$ is injective for every $E_{j}$ (e.g. $E_{j}$ projective) and $E$ is pure in $\Pi E_{j}$, then $f$ is injective. Theorem. If $R$ is a Dedekind domain and $E$ is flat then $f$ is injective for $E$ iff $f$ is injective for $\operatorname{Hom}(F$, $E$ ) for all modules $F$. Theorem. If $R$ is a Dedekind domain and $f$ is injective for $E$ for all families $\left(F_{i}\right)$ then $E$ is reduced. ThEOREM. If $R$ is commutative and $f$ is always injective then $R$ must be artinian. The converse holds for serial rings.

Introduction. If $E$ is a right module and $\left(F_{i}\right)_{i \in I}$ is a family of left modules over an associative ring $R$ with 1 then it is always true that the groups $E \otimes_{R}\left(\bigoplus_{i \in I} F_{i}\right)$ and $\bigoplus_{i \in I}\left(E \otimes_{R} F_{i}\right)$ are isomorphic. However, it is not hard to see that the groups $E \otimes_{R} \Pi_{i \in I} F_{i}$ and $\Pi_{i \in I}\left(E \bigotimes_{R} F_{i}\right)$ are not necessarily isomorphic (e.g. if $R$ is the integers let $E$ be the rationals, $I$ the natural numbers and, for a fixed prime $p, F_{n}$ the cyclic group of order $p^{n}$. This example is found in ([8], p. 257, Ex. 10).

It is our purpose to study the relationship between the groups $E \otimes_{R} \prod_{i \in I} F_{i}^{\prime}$ and $\prod_{i \in I}\left(E \otimes_{R} F_{i}\right)$. We will do so in terms of the natural homomorphism

$$
f: E \bigotimes_{R} \prod_{i \in I} F_{i} \longrightarrow \prod_{i \in I}\left(E \bigotimes_{R} F_{i}\right)
$$

which sends a generator $x \otimes\left(y_{i}\right)_{i \in I}$ of $E \bigotimes_{R} \prod_{i \in I} F_{i}$ onto $\left(x \otimes y_{i}\right)_{i \in I}$ in $\Pi_{i \in I}\left(E \otimes_{R} F_{i}\right)$.

In $\S \S 1$ and 2 we investigate the properties of $f$ over an arbitrary ring. It is relatively easy to show that $f$ is surjective (bijective) for all families $\left(F_{i}\right)_{i \in I}$ if and only if $E$ is finitely generated (finitely presented). In $\$ \S 3$ and 4 we study the more difficult problem of when $f$ is injective. In $\S 3$ the ring is a Dedekind domain. In $\S 4$ the ring is commutative artinian.

We will always denote by $R$ an associative ring with 1 , by $E$ a right $R$-module, and by $\left(F_{i}\right)_{i \in I}$ a family of left $R$-modules. Modules 
are all unitary.

We will often use the notions of flatness and purity. Briefly, a left $R$-module $F$ is said to be flat if the functor ( $) \bigotimes_{R} F$ is exact. A submodule $E^{\prime}$ of a right $R$-module $E$ is said to be pure if ( ) $\otimes_{R} F$ is exact on $0 \rightarrow E^{\prime} \stackrel{\text { incl }}{\longrightarrow} E \stackrel{\text { proj }}{\longrightarrow} E / E^{\prime} \rightarrow 0$ for every right module $F$.

Over a domain it is well known that torsion free modules are flat iff the domain is Prüfer. It is also known that over a domain, $E^{\prime}$ is pure in $E$ means $r E^{\prime}=E^{\prime} \cap r E$ for all $r \in R$ iff the domain is Prüfer. For this last fact see [13].

Many of our arguments involve a diagram chase. At these points we usually will be able simply to cite the following.

Snake Lemma [2, Ch. 1, 1 , No. 4, Prop. 2]. Suppose

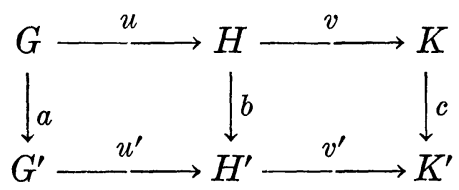

is a commutative diagram of abelian groups with exact rows. Then this diagram can be embedded in the following commutative diagram with top and bottom row not necessarily exact.

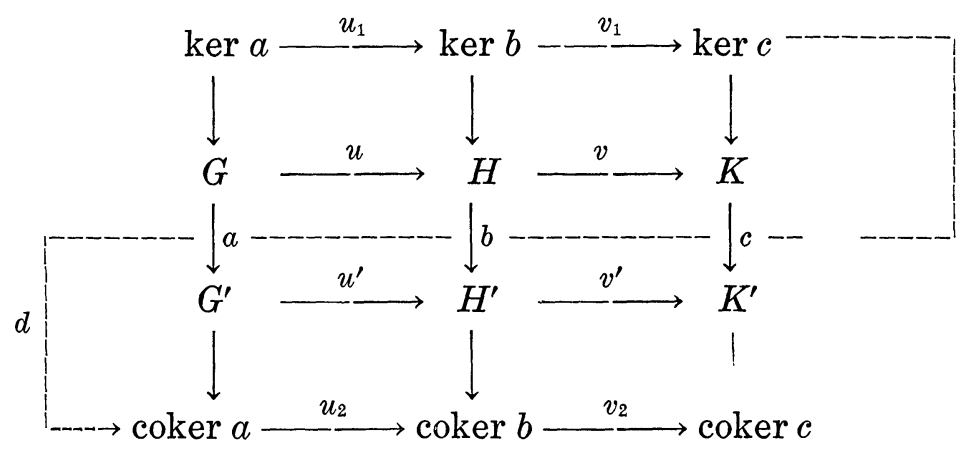

The following are true of this diagram

(i) $v_{1}^{\circ} u_{1}=0$. If $u^{\prime}$ is injective then the top row is exact.

(ii) $v_{2} \circ u_{2}=0$. If $v$ is surjective the bottom row is exact.

(iii) If $u^{\prime}$ is injective and $v$ is surjective there exists a unique homomorphism $d$ such that the sequence $\left(u_{1}, v_{1}, d, u_{2}, v_{2}\right)$ is exact.

For convenience we will omit writing the indexing set whenever there is no possibility of confusion. For example, we will write $\Pi F_{i}$, $\bigoplus F_{i}$ and $\left(F_{i}\right)$, omitting the index set $I . f g$ and $f p$ will denote, respectively, finitely generated and finitely presented. Instead of writing $E \otimes{ }_{R} F$ we will simply write $E \otimes F$. Often an obvious homomorphism will not be defined explicitly or even named. $Z$ will be the ring of integers, $Q$ the field of rational numbers, and, for a positive integer 
$n, Z(n)$ will denote the cyclic group of order $n$.

This study is a doctoral dissertation prepared under the direction of Professor Edgar Enochs and submitted to the University of Kentucky.

\section{Surjectivity and bijectivity.}

Proposition 1.1. If $E$ is $f g$ and projective then $f$ is bijective.

Proof. If $E^{\prime}$ is $f g$ and free then for some positive integer $n$, $E^{\prime} \approx R^{n}$. We have the canonical isomorphisms

$$
E^{\prime} \otimes \Pi F_{i} \approx R^{n} \otimes \prod F_{i} \approx\left(\Pi F_{i}\right)^{n}
$$

and

$$
\Pi\left(E^{\prime} \otimes F_{i}\right) \approx \Pi\left(R^{n} \otimes F_{i}\right) \approx \Pi F_{i}^{n} .
$$

So $f$ is just the canonical isomorphism $\left(\Pi F_{i}\right)^{n} \approx \Pi F_{i}^{n}$.

If $E$ is $f g$ and projective then $E$ is a direct summand of such an $E^{\prime}$. We show later (Prop. 2.1) that if $f$ is injective for $E^{\prime}$ then $f$ is injective for any summand of $E^{\prime}$.

Proposition 1.2. $f$ is surjective (for all families $\left(F_{i}^{\prime}\right)$ ) iff $E$ is $f g$.

Proof. Suppose $E$ is $f g, n$ is a positive integer, and $R^{n} \rightarrow E \rightarrow 0$ is exact. Consider the commutative diagram with exact rows

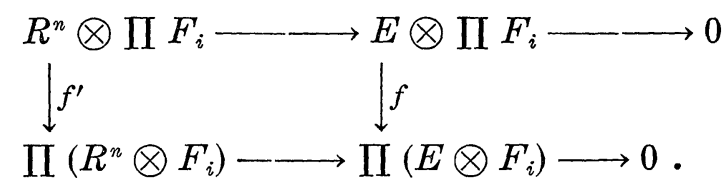

$f^{\prime}$ is bijective (Prop. 1.1). The snake lemma tells us $f$ is surjective.

On the other hand, suppose $E$ is not $f g$. Let $I=E$ ( $E$ considered as a set), $F_{i}={ }_{R} R$. Write $E=\left\{x_{i}\right\}$. Then $\left(x_{i} \otimes 1\right)_{i \in I} \in \Pi\left(E \otimes F_{i}\right)$ is not in imf. So $f$ is not surjective.

REMARK 1. In order to show $E f g$ all we need to show is that $f$ is surjective for all families $\left(F_{i}\right)$ with $F_{i}={ }_{R} R$.

Remark 2. The part of Proposition 1.2 that supposes $E f g$ is found in [3, Ch. II, Ex. 2].

If $g$ and $h$ are injective homomorphisms of right, respectively, left $R$-modules, having domains $E$ and $F$, respectively, we denote $\operatorname{im}(g \otimes h)$ as $[E \otimes F]$.

Proposition 1.3. $f$ is bijective (for all families $\left(F_{i}\right)$ ) iff $E$ is $f p$. 
Proof. Suppose $E$ is $f p$ and $0 \rightarrow K \rightarrow L \rightarrow E \rightarrow 0$ is a finite presentation of $E$. Consider the commutative diagram with exact rows

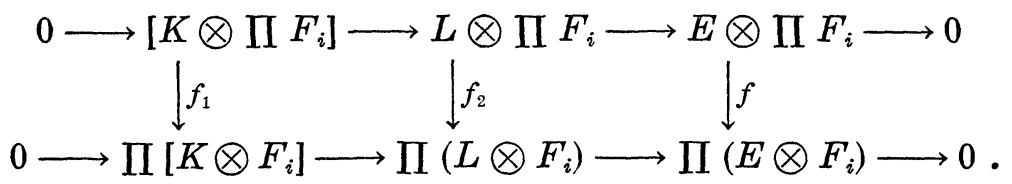

$f_{1}$ is surjective because $K$ is $f g$ (Prop. 1.2). $f_{2}$ is bijective because $L$ is $f g$ and free (Prop. 1.1). By the snake lemma, therefore, $f$ is bijective.

On the other hand, suppose $f$ is bijective for all families $\left(F_{i}\right)$. Since $f$ is surjective, $E$ is $f g$ (Prop. 1.2). So we have an exact sequence $0 \rightarrow K \rightarrow L \rightarrow E \rightarrow 0$ where $L$ is $f g$ and free. By Remark 1 following Prop. 1.2 to show $K f g$ it suffices to show $f$ is surjective for $K$ for all families $\left(F_{i}\right), F_{i}={ }_{R} R$.

For such a family consider the commutative diagram with exact rows

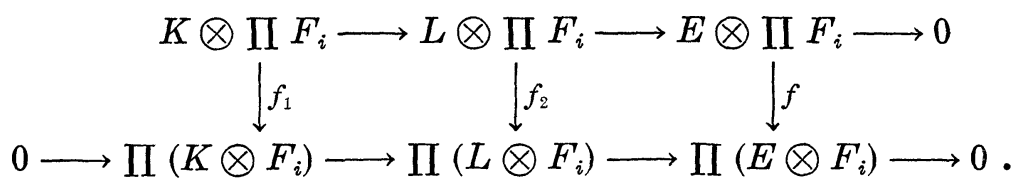

$f_{2}$ is bijective because $L$ is $f g$ and free (Prop. 1.1). $f$ is bijective by assumption. Therefore, by the snake lemma, $f_{1}$ is surjective.

REMARK 1. It is found in [3, Ch. II, Ex. 2] that if $R$ is right noetherian and $E f g$ then $f$ is bijective. More generally, [2, Ch. 1, Ex. 8] states that if $E$ is $f p$ then $f$ is bijective.

REMARK 2. The first half of the proof of Prop. 1.3 shows that if $f$ is injective for $E$ then $f$ is injective for any quotient of $E$ by a $f g$ submodule.

\section{Injectivity.}

Proposition 2.1. Suppose $J$ is a set and $E_{j}$ is a right $R$-module for every $j \in J$. Let $E \approx \oplus E_{j}$. Then $f$ is injective for $E$ iff $f$ is injective for every $E_{j}$. If $E^{\prime}$ is pure in $E$ and $f$ is injective for $E$ then $f$ is injective for $E^{\prime}$.

Proof. It is easy to see that if $J$ is finite and $f$ is injective for every $E_{j}$ then $f$ is injective for $E$. Suppose $J$ is infinite,

$$
z=\sum_{i=1}^{n} x_{\lambda} \otimes y_{\lambda} \in E \otimes \Pi F_{i},
$$


and $f(z)=0$. Let $J^{\prime}$ be the support of $\left\{x_{\lambda}\right\}_{\lambda=1}^{n}$. Consider the commutative diagram

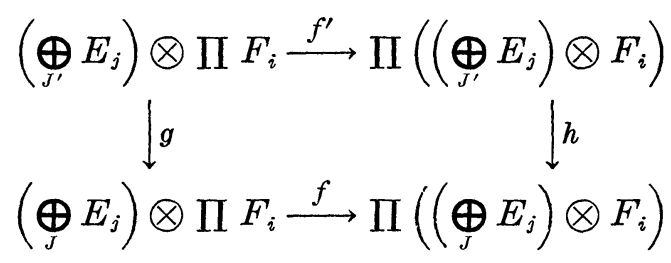

where $g$ and $h$ are the obvious injective homomorphisms. If $\boldsymbol{z}^{\prime}=$ $\sum_{\lambda=1}^{n} x_{\lambda} \otimes y_{\lambda} \in\left(\bigoplus_{J^{\prime}} E_{j}\right) \otimes \prod F_{i}$ then $g\left(z^{\prime}\right)=z$. But since $J^{\prime}$ is finite, $f^{\prime}$ is injective. Therefore $z^{\prime}=0$, hence $z=0$. So $f$ is injective.

For the remainder of the proposition suppose $f$ is injective for $E$ and $E^{\prime}$ is pure in $E$. Consider the commutative diagram with exact rows

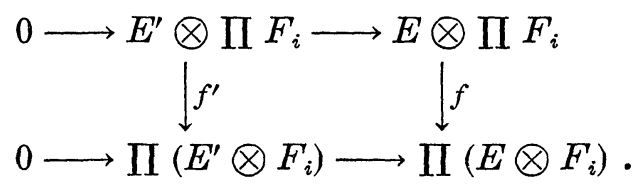

Since $f$ is injective the snake lemma says $f^{\prime}$ is injective.

REMARK. It is not hard to see that if $E^{\prime}$ is a pure submodule of $E$ and $f$ is injective for $E^{\prime}$ and $E / E^{\prime}$ then $f$ is injective for $E$. Merely apply the snake lemma to the following commutative diagram with exact rows

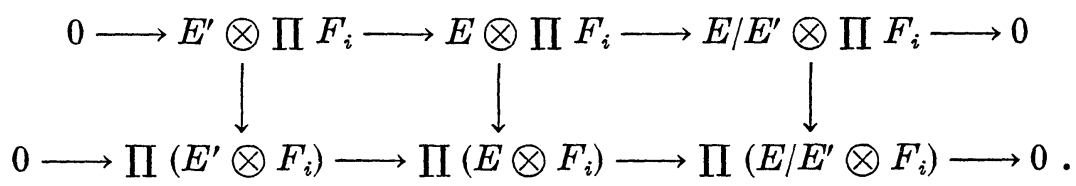

Proposition 2.2. If $E$ is the quotient of a projective by a $f g$ submodule then $f$ is injective.

Proof. If $E$ is free and $E \approx \oplus R$ then we have the canonical isomorphisms

$$
E \otimes \Pi F_{i} \approx(\oplus R) \otimes \Pi F_{i} \approx \oplus \Pi F_{i}
$$

and

$$
\Pi\left(E \otimes F_{i}\right) \approx \Pi\left(\oplus R \otimes F_{i}\right) \approx \Pi \oplus F_{i} .
$$

So $f$ is the injection $\oplus \Pi F_{i} \rightarrow \Pi \oplus F_{i}$.

If $E$ is projective then $E$ is a summand of a free module. Hence 
$f$ is injective for $E$ (Prop. 2.1). The proposition now follows from Remark 2 following Prop. 1.3.

We want to use the following result of Matlis.

THEOREM 2.3. (Matlis, [12].) If $R$ is right noetherian and $E$ is injective then $E$ is the direct sum of indecomposable, injective submodules.

It will be useful to consider $f$ when every $F_{i}$ of the family $\left(F_{i}\right)$ is flat. Such a family will be called a flat family.

Proposition 2.4. $f$ is injective for all flat families $\left(F_{i}\right)$ iff $R$ is right noetherian and $f$ is injective for all indecomposable injective $E$ for all flat families $\left(F_{i}\right)$.

Proof. Suppose $f$ is injective for all flat families $\left(F_{i}\right)$ and $E$ is a right ideal of $R$. Recall that to show $E$ is $f g$ it suffices to show $f$ is surjective for $E$ for all families $\left(F_{i}\right)$ with $F_{i}={ }_{R} R$ (Remark 1, following Prop. 1.2). So suppose $\left(F_{i}\right)$ is such a family. Consider the commutative diagram with exact rows

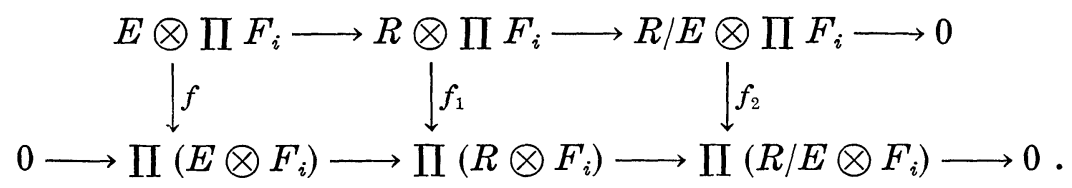

$f_{1}$ is bijective. $f_{2}$ is injective by supposition. By the snake lemma $f$ is surjective.

For the converse, suppose $\left(F_{i}\right)$ is a flat family and $E^{\prime}=\oplus E_{j}$ is the injective envelope of $E$ where $E_{j}$ is indecomposable injective. By Prop. 2.1, $f$ is injective for $E^{\prime}$. Consider the commutative diagram with exact rows

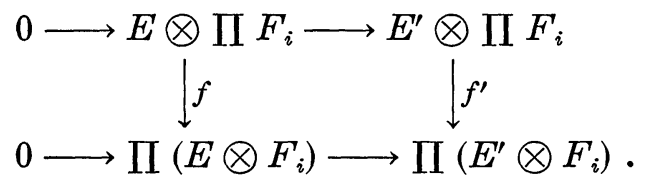

(The top row is exact because over a right noetherian ring the direct product of flat left modules is flat, [3, Ch. VI, Ex. 4].) Since $f^{\prime}$ is injective the snake lemma says $f$ is also injective.

3. Dedekind domains. Let $K$ be the field of fractions of a domain $R$. If $R$ is a Dedekind domain the indecomposable injective $R$-modules are $K$ and, for primes $P$, the $P$-primary components of $K / R$ [9, Thm. 7]. Adopting the notation that is standard when $R$ 
is the ring of integers we will denote the $P$-primary component of $K / R$ as $R\left(P^{\infty}\right)$.

In order to establish that Dedekind domains possess the property of Prop. 2.4 we prove the following lemma.

LEMMA 3.1. If $S$ is a multiplicative subset of an arbitrary commutative ring $R, E=S^{\sim 1} R$ and $\left(F_{i}\right)$ is a family of torsion free $R$-modules then $f$ is injective.

Proof. If $0 \in S$ then $S^{-1} R=0$. So assume $0 \notin S$. We have the canonical isomorphisms [2, Ch. II, §2, no. 7, Prop. 18], $S^{-1} R \otimes \Pi F_{i} \approx$ $S^{-1}\left(\Pi F_{i}\right)$ and $\Pi\left(S^{-1} R \otimes F_{i}\right) \approx \Pi\left(S^{-1} F_{i}\right)$. So we can think of $f$ as the canonical map $S^{-1}\left(\Pi F_{i}\right) \longrightarrow \Pi\left(S^{-1} F_{i}\right)$. Suppose $f\left(\left(y_{i}\right) / s\right)=\left(y_{i} / s\right)=0$. Then for every $i \in I$ there exists $t_{i} \in S$ such that $t_{i} y_{i}=0$. Since $t_{i} \neq 0$, $y_{i}=0$. Hence $f$ is injective.

Proposition 3.2. If $R$ is a Dedekind domain then $f$ is injective for all flat families $\left(F_{i}\right)$.

Proof. By Prop. 2.4 and Prop. 2.1 it suffices to show $f$ is injective for $K$ and $K / R$. But since we have the following commutative diagram with exact rows

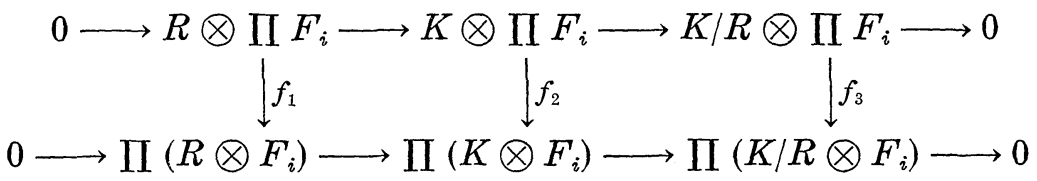

where $f_{1}$ is bijective we know from the snake lemma that $f_{2}$ is injective iff $f_{3}$ is injective. That $f_{2}$ is injective is Lemma 3.1. The proof is now complete.

Since a Prüfer domain is Dedekind iff it is noetherian we have an immediate corollary.

COROLlary 3.3. If $R$ is a Prüfer domain then $f$ is injective for all flat families $\left(F_{i}\right)$ iff $R$ is Dedekind.

There is an interesting generalization of Prop. 3.2. We need the following lemma whose proof is immediate from the definitions.

Lemma 3.4. If $R$ is a Prüfer domain and $\left(F_{i}, g_{i}^{j}\right)$ is an inverse system of flat $R$-modules then $\lim F_{i}$ is a pure submodule of $\Pi F_{i}$.

Theorem 3.5. If $R$ is a Dedekind domain and $\left(F_{i}, g_{i}^{j}\right)$ is an 
inverse system of flat $R$-modules then the canonical homomorphism

$$
F: E \otimes \lim _{\longleftarrow} F_{i} \longrightarrow \lim _{\longleftarrow}\left(E \otimes F_{i}\right)
$$

is injective.

Proof. Clearly $\left(E \otimes F_{i}\right.$, id $\left.\otimes g_{i}^{j}\right)$ is an inverse system and $F$ is a homomorphism. To show $F$ injective consider the commutative diagram

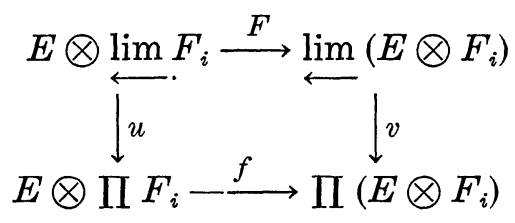

where $u$ and $v$ are the obvious maps. $u$ is injective (Lemma 3.4). $f$ is injective (Prop. 3.2). By the Snake lemma $F$ must also be injective.

REMARK. Suppose $R$ is any ring for which $f$ is injective for all flat families $\left(F_{i}\right)$. Then it is easy to see that $F$ is injective for the inverse system of flat left modules $\left(F_{i}\right)$ iff $\lim ^{\longleftarrow} F_{i}$ is pure in $\Pi F_{i}$. Just chase the following commutative diagram

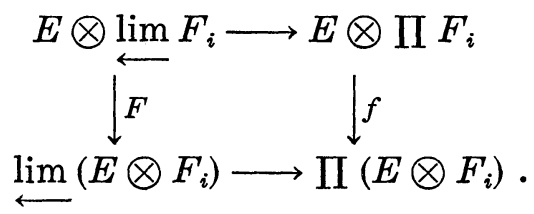

In Prop. 2.2 we established that $f$ is injective whenever $E$ is projective. We will see later (Thm. 3.13) that this cannot be extended to the case when $E$ is flat. But when $R$ is a Dedekind domain we do have the following generalization.

Theorem 3.6. If $R$ is a Dedekind domain, $\left(E_{j}\right)_{j \in J}$ is a family of flat $R$-modules such that $f$ is injective for every $E_{j}$ (e.g. $E_{j}$ is projective), and $E$ is isomorphic to a pure submodule of $\Pi E_{j}$, then $f$ is injective for all families $\left(F_{i}\right)$.

Proof. Consider the following diagram where all maps are the obvious ones 


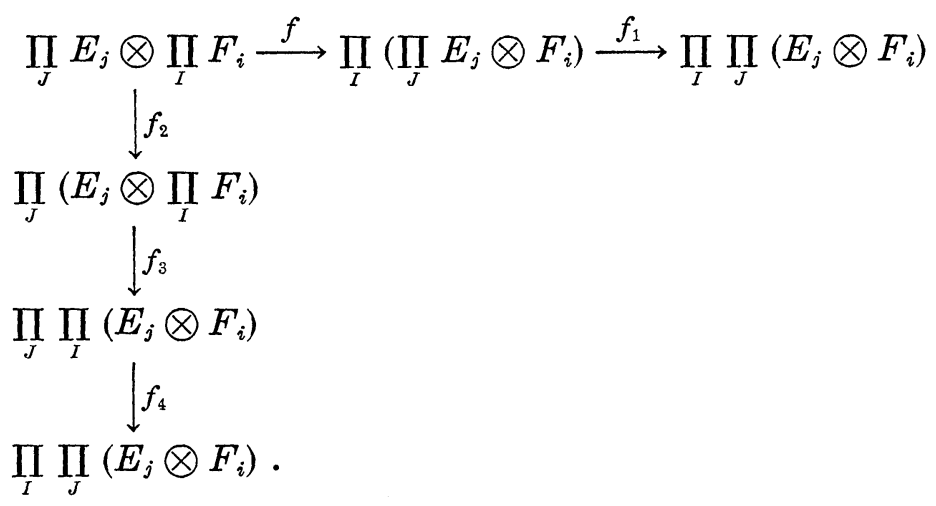

$f_{1}$ and $f_{2}$ are injective (Prop. 3.2) and $f_{3}$ is injective by hypothesis. Therefore, $f_{4} \circ f_{3} \circ f_{2}=f_{1} \circ f$ is injective. Hence $f$ is injective. Now apply Prop. 2.1.

REMARK 1. We have not used any specific property of Dedekind domains in Thm. 3.6. The theorem holds for any ring for which $f$ is injective for flat families $\left(F_{i}\right)$.

REMARK 2. If in Thm. 3.6, $J$ is infinite then $\Pi_{J} Z$ is not free (e.g. [8, Thm. 19.2]). Indeed it is not hard to see that in any presentation of $\Pi_{J} Z$ there are infinitely many defining relations [8, p. 95, Ex. 6]. Thus Thm. 3.6 genuinely enlarges the class of modules $E$ as described in Prop. 2.2 for which $f$ is injective.

CoRollary 3.7. If $R$ is a Dedekind domain, $\left(E_{j}\right)_{j \in J}$ is an inverse system of flat $R$-modules such that $f$ is injective for every $E_{j}$ (e.g. $E_{j}$ is projective), and $E=\lim _{\longleftarrow} E_{j}$ then $f$ is injective.

Proof. By Lemma 3.4, $\lim E_{j}$ is pure in $\Pi E_{j}$. Now apply Thm. 3.6.

There is a characterization of modules $E$ for which $f$ is injective in terms of groups of homomorphisms. First a crucial result of D. Lazard.

Theorem 3.8. (Lazard, [11].) A right $R$-module $E$ is flat iff $E$ is the direct limit of $f g$ free modules.

Definition 3.9. If $R$ is commutative and $E$ and $F$ are $R$-modules we will call the module $\operatorname{Hom}_{R}(E, F)$ the $F$-dual of $E$.

The $R$-dual of $E$ is commonly called, simply, "the dual of $E$ ". Henceforth we will omit the $R$ when writing $\operatorname{Hom}_{R}(E, F)$. 
Theorem 3.10. If $R$ is a Dedekind domain and $E$ is a flat $R$ module then $f$ is injective for $E$ iff $f$ is injective for all $E$-duals.

Proof. If $f$ is injective for all $E$-duals then $f$ is injective for the $E$-dual of $R$, $\operatorname{Hom}(R, E) \approx E$.

Suppose, on the other hand, that $f$ is injective for $E$. To show $f$ is injective for all $E$-duals it suffices to consider the $E$-duals of flat modules since if $T$ is the torsion submodule of an arbitrary module $F$ then $\operatorname{Hom}(F, E) \approx \operatorname{Hom}(F / T, E)$. But if $F$ is flat then $F \approx \lim F_{j}$ where $F_{j}$ is $f g$ and free, say of rank $n_{j}$. Therefore, Hom $(F, \vec{E}) \approx$ $\operatorname{Hom}\left(\lim F_{j}, E\right) \approx \lim \operatorname{Hom}\left(F_{j}, E\right)$ where $\operatorname{Hom}\left(F_{j}, E\right) \approx E^{n_{j}}$. Since $f$ is injective for $\overleftrightarrow{\operatorname{Hom}}\left(F_{j}, E\right)$ (Prop. 2.1) and $\operatorname{Hom}\left(F_{j}, E\right)$ is flat, $f$ is injective for $\lim \operatorname{Hom}\left(F_{j}, E\right)$ (Cor. 3.7). Hence $f$ is injective for $\operatorname{Hom}(F, E)$.

REMARK 1. Suppose $R$ is a Dedekind domain, $\left(E_{j}\right)$ is family of flat modules, $E^{\prime}$ is a pure submodule of the flat module $E$, and $f$ is injective for $E$ and every $E_{j}$. Then (a) $f$ is injective for $\oplus E_{j}$ (Prop. 2.1), (b) $f$ is injective for $\Pi E_{j}$ (Thm. 3.6), and (c) $f$ is injective for $E^{\prime}$ (Prop. 2.1). By Thm. 3.10 we also know (a') $f$ is injective for all $\oplus E_{j}$-duals, $\left(\mathrm{b}^{\prime}\right) f$ is injective for all $\Pi E_{j}$-duals, and $\left(\mathrm{c}^{\prime}\right) f$ is injective for all $E^{\prime}$-duals. ((a) and $\left(a^{\prime}\right)$ are actually special cases of (c) and $\left(\mathrm{c}^{\prime}\right)$ respectively.) It is perhaps worthwhile to note that $\left(a^{\prime}\right),\left(b^{\prime}\right)$, and $\left(c^{\prime}\right)$ can be proved directly by noting the following where $F \approx \lim F_{\lambda}$ is an arbitrary flat module, $F_{\lambda}$ is free and of finite rank $n_{\lambda}$.

(i) $\operatorname{Hom}\left(F, \oplus E_{j}\right)$ is pure in $\operatorname{Hom}\left(F, \Pi E_{j}\right)$.

(ii) $f$ is injective for $\operatorname{Hom}\left(F, \Pi E_{j}\right) \approx \Pi \operatorname{Hom}\left(F, E_{j}\right) \approx \Pi \lim E_{j}^{n_{\lambda}}$ by Cor. 3.7 and Thm. 3.6.

(iii) $\operatorname{Hom}\left(F, E^{\prime}\right) \approx \lim \left(E^{\prime}\right)^{n_{\lambda}}$ is pure in $\operatorname{Hom}(F, E) \approx \lim E^{n_{\lambda}}$ and $f$ is injective for $\lim E^{n_{\lambda}}$ by Cor. 3.7.

REMARK 2. It is known that the $Z$-dual of a countable direct product of copies of $Z$ is free [6]. Thus $E=\operatorname{Hom}(F, Z)$ when $F$ is such a product does not enlarge beyond our previous knowledge our class of flat groups $E$ for which $f$ is injective.

In general when $E$ is flat and $f$ is injective for $E$ it seems largely unknown what the $E$-duals are. $\left(\operatorname{Hom}\left(\Pi^{\infty} Z, Z\right) \approx \oplus^{\infty} Z\right.$ is the only example we know.) Thus it is unclear how and if Thm. 3.10 does in fact enlarge the class of flat groups $E$ for which $f$ is injective. The identity of such $E$-duals seems a matter of general and independent interest. 
It is an easy consequence of [3, Ch. I, Ex. 8] that any right module over a right noetherian ring possesses a maximal injective submodule. The complement of this maximal injective submodule has no injective submodules. Therefore, over a Dedekind domain, an arbitrary module $E$ can be written as a direct sum $E_{1} \oplus E_{2} \oplus E_{3}$ where $E_{1}$ is a direct sum of $R\left(P^{\infty}\right)^{\prime}$ 's, $E_{2}$ is a direct sum of quotient fields $K$, and $E_{3}$ has no injective submodules. Since injectivity $\equiv$ divisibility over a Dedekind domain [9, Thm. 6], it is consistent with terminology in abelian groups to say $E_{3}$ is reduced. We claim that if $f$ is injective for $E$ then $E$ is reduced, i.e., $E_{1} \oplus E_{2}=0$. We need the following lemmas.

Lemma 3.11. If $R$ is a Dedekind domain, $P$ is a prime ideal and $a \in R-P$ then $R / P^{n}$ is a-divisible for every positive integer $n$.

Proof. Define $\left(P^{n}: a\right)=\left\{b \in R: b a \in P^{n}\right\}$. To show

$$
g: R / P^{n} \longrightarrow R / P^{n}
$$

given by $b+P^{n} \longrightarrow a b+P^{n}$ is bijective note that $\left(P^{n}: a\right)=P^{n}$. Thus $g$ is injective. But $R / P^{n}$ has a (finite) composition series. Hence $g$ must be surjective.

LemMa 3.12. If $R$ is a Dedekind domain with prime ideal $P$, $F=\prod_{n=1}^{n}\left(R / P^{n}\right)$, and $h$ is the canonical homomorphism $R \otimes F \rightarrow K \otimes$ $F$ then $h$ is not surjective.

Proof. Note that $K \otimes F$ is divisible. If im $h$ were divisible then for an arbitrary $0 \neq p \in P$ there would exist $1 \otimes\left(b_{n}\right)_{n=1}^{\infty} \in R \otimes F$ such that $1 \otimes\left(1-p b_{n}\right)_{n=1}^{\infty} \in \operatorname{ker} h$. But ker $h$ is torsion [3, Ch. VII, Prop. 4.6]. (One can see this directly.) Thus there is $0 \neq t \in R$ such that $t\left(1-p b_{n}\right)=0$ for all $n$. Setting $x_{n}=1-p b_{n}^{\prime}$ where $b_{n}^{\prime} \in R$ and $b_{n}^{\prime}+$ $P^{n}=b_{n}$, we have $t x_{n} \in P^{n}$. Let $m$ be the largest integer such that $t \in P^{m}$. If $t \notin P$, let $m=0$. We claim $x_{m+1} \in P$.

If not there is $y \in R$ such that $x_{m+1} y-1=s \in P$. So $y x_{m+1} t=$ $(1+s) t=t+s t \in P^{m+1}$. Thus $t \in P^{m+1}$. Contradiction.

Since $x_{m+1}=1-p b_{m+1}^{\prime}, 1=x_{m+1}+p b_{m+1}^{\prime} \in P$. Contradiction. So im $h$ could not have been divisible.

Theorem 3.13. If $R$ is a Dedekind domain and $f$ is injective for $E$ then $E$ must be reduced.

Proof. By Prop. 2.1 it suffices to show for a given prime ideal $P$ there exists a family $\left(F_{i}\right)$ such that $f$ is not injective for $K$ and $f$ is not injective for $R\left(P^{\infty}\right)$. We do this by choosing $F_{n}=R / P^{n}, n=$ 
$1,2, \cdots$, and showing $K \otimes F_{n}=R\left(P^{\infty}\right) \otimes F_{n}=0$ but $K \otimes \prod_{n=1}^{\infty} F_{n} \neq$ $0 \neq R\left(P^{\infty}\right) \otimes \prod_{n=1}^{\infty} F_{n}$.

Since $R\left(P^{\infty}\right)$ and $K$ are divisible, $R\left(P^{\infty}\right) \otimes F_{n}=K \otimes F_{n}=0$.

Set $F=\prod_{n=1}^{\infty} F_{n}$ and denote by $T$ the torsion submodule of $F$. We have the exact sequence

$$
K \otimes F \longrightarrow K \otimes F / T \longrightarrow 0
$$

where $K$ and $F / T$ are nonzero flat modules. Since $K \otimes F / T \neq 0, K \otimes$ $F \neq 0$ by exactness.

Suppose $Q$ is a prime different from $P, m$ is a positive integer and $a \in Q^{m}-P$. Then $F_{n}$ is $a$-divisible for every $n$ (Lemma 3.11). So $F$ is $a$-divisible. Hence $R\left(Q^{\infty}\right) \otimes F=0$. Therefore, $R\left(P^{\infty}\right) \otimes F \approx$ $K / R \otimes F$.

To show $K / R \otimes F \neq 0$ consider the exact sequence

$$
R \otimes F \stackrel{h}{\longrightarrow} K \otimes F \longrightarrow K / R \otimes F \longrightarrow 0
$$

where $h$ is the obvious map. Lemma 3.12 says $h$ is not surjective. By exactness $K / R \otimes F \neq 0$.

REMARK. Over a Dedekind domain a reduced module that is not flat is either (1) a direct sum of a flat module and a finite direct sum of modules of type $R / P^{n}$ where $P$ is prime, or (2) has an infinite proper chain of direct summands each summand itself a finite direct sum of modules of type $R / P^{n}$ [9, Thm. 9].

Since $f$ is injective for any module of type $R / P^{n}$ (Prop. 1.3), injectivity for modules of type (1) reduces to injectivity of reduced flat modules. Although we have some important examples of reduced flat modules for which $f$ is injective, $f$ need not be injective for such a module. This is shown by Prop. 3.15.

It is also true that $f$ need not be injective for modules of type (2). This is shown by Prop. 3.14.

Proposition 3.14. Suppose $p$ is a prime integer and

$$
E=\Pi_{n=1}^{\infty} Z\left(p^{n}\right) \text {. }
$$

Then $f$ is not injective for $E$. (Obviously $E$ is of type (2).)

Proof. Choose a prime $q$ different from $p$. Let $F=\prod_{n=1}^{\infty} Z\left(q^{n}\right)$ and denote by $S$ and $T$ the torsion subgroups of $E$ and $F$ respectively. Consider the exact sequence

$$
E \otimes F \longrightarrow E / S \otimes F / T \longrightarrow 0 .
$$

Since $E / S$ and $E / T$ are nonzero and flat, $E / S \otimes F / T \neq 0$. By exact- 
ness, $E \otimes F \neq 0$.

To complete the proof note that $E \otimes Z\left(q^{n}\right)=0$ for every integer $n$.

REMARK. It is easy to check that $\Pi Z(p)$ where $p$ ranges over the primes is another group of type (2) for which $f$ is not injective.

Proposition 3.15. Suppose $E$ is a reduced torsion free abelian group that is divisible for a prime integer $p$. Then $f$ is not injective for $E$.

Proof. Since $E$ is $p$-divisible, $E \otimes Z\left(p^{n}\right)=0$ for all positive n. Therefore $\prod_{n=1}^{\infty}\left(E \otimes Z\left(p^{n}\right)\right)=0$.

If $F=\prod_{n=1}^{\infty} Z\left(p^{n}\right)$ and $T$ is the torsion subgroup of $F$ consider the exact sequence

$$
E \otimes F \longrightarrow E \otimes F / T \longrightarrow 0 .
$$

Since $E$ and $F / T$ are nonzero and torsion free $E \otimes F / T \neq 0$. By exactness, $E \otimes F \neq 0$.

$$
\begin{gathered}
\text { Since } E \otimes \prod_{n=1}^{\infty} Z\left(p^{n}\right) \neq 0 \text { and } \prod_{n=1}^{\infty}\left(E \otimes Z\left(p^{n}\right)\right)=0, \\
f: E \otimes \prod_{n=1}^{\infty} Z\left(p^{n}\right) \longrightarrow \prod_{n=1}^{\infty}\left(E \otimes Z\left(p^{n}\right)\right)
\end{gathered}
$$

is not injective.

REMARK 1. There are many examples of reduced torsion free groups that are divisible for a prime $p$. For a prime $q$ different from $p$, the $q$-adic integers are such a group. The integers localized at $q, Z_{q}$, is another common example.

Concerning $Z_{q}$, if $E$ is any torsion free group of rank 1 whose type is $\left(k_{1}, k_{2}, \cdots\right)$, then $E$ is reduced and divisible for $p$ iff the $k_{n}$ corresponding to $p$ is $\infty$ but at least one other $k_{n}$ is finite. (For the notion of type and a discussion of torsion free groups of rank 1 see [7].)

REMARK 2. We do not know if for every prime integer $p, E$ is not $p$-divisible then $f$ is injective. However, we have since shown this is true for rank 1 torsion free groups.

We have following interesting corollary to Thm. 3.13.

CoRollary 3.16. If $R$ is a Dedekind domain and $\left(F_{i}\right)$ is a flat family such that $E \otimes F_{i}$ is reduced then $E \otimes \Pi F_{i}$ is reduced.

Proof. $\Pi\left(E \otimes F_{i}\right)$ is reduced and $f$ is injective (Prop. 3.2). Therefore $E \otimes \Pi F_{i}$ is reduced. 
REMARK. A special case is when $E$ is reduced. Then $E \otimes \Pi R$ is reduced where the product is arbitrary

4. Commutative artinian rings. Matlis [12, Thm. 3.11] has shown that over a commutative artinian ring all indecomposable injective modules are $f g$. And we have seen that $f$ is injective for all flat families $\left(F_{i}\right)$ iff $R$ is right noetherian and $f$ is injective for all flat families $\left(F_{i}\right)$ whenever $E$ is indecomposable injective (Prop. 2.4). Therefore, by Prop. 1.3 we know the following

THEOREM 4.1. Over a commutative artinian ring $f$ is injective for all flat families $\left(F_{i}\right)$.

REMARK 1. It is possible to prove this theorem by purely elementary methods, without resorting to Matlis' result.

REMARK 2. Thm. 4.1 endows commutative artinian rings with the property that was necessary to prove Thm. 3.6. (See Remark 1, following that theorem.) We have, therefore, the following

THEOREM 4.2. If $R$ is commutative artinian, $\left(E_{j}\right)$ is a family of flat modules, $f$ is injective for $E_{j}$, and $E$ is a pure submodule of $\Pi E_{j}$, then $f$ is injective for all families $\left(F_{i}\right)$.

REMARK. Thm. 4.2 can be derived in a different way by noting that over a commutative artinian ring every flat module is projective [1] and that the direct product of projective modules is projective [4].

We do not know that if $R$ is commutative artinian then $f$ is always injective. But if $f$ does have this property $R$ must be commutative artinian. To prove this we need two lemmas. A multiplicative subset of $R$ will always be assumed to possess 1 and not 0 . If $r \in R$ then $(r)$ will denote the ideal generated by $r$.

LEMma 4.3. Suppose $S$ is a multiplicative subset of the commutative ring $R$. Then

$$
S \cap\left(\bigcap_{s \in S}(s)\right) \neq \varnothing \text { iff } S^{-1} R \otimes \prod_{s \in S} R /(s)=0 .
$$

Therefore, if $f$ is injective we have $S \cap\left(\bigcap_{s \in S}(s)\right) \neq \varnothing$ for all multiplicative subsets $S$ of $R$.

Proof. For convenience we will write $R /(s)$ as $\bar{s}$ and omit the index set of products and intersections when this set is obviously $S$. Note that $S^{-1} R \otimes \Pi \bar{s} \approx S^{-1}(\Pi \bar{s})$, naturally. 
If $t \in S \cap(\cap(s))$ and $x / u \in S^{-1}(\Pi \bar{s})$ then $x / u=t x / t u=0 / t u=0$. So $S \cap(\cap(s)) \neq \varnothing$ implies $S^{-1} R \otimes \Pi \bar{s}=0$.

If, on the other hand, $S^{-1} R \otimes \Pi \bar{s}=0$ then $(1+(s)) s \in S / 1=0$ in $S^{-1}(\Pi \bar{s})$. So there exists $t \in S$ such that $t \in \cap(s)$. Hence

$$
S \cap(\cap(s)) \neq \varnothing \text {. }
$$

For the rest, note that for $s \in S, S^{-1} R \otimes \bar{s}=0$. So $\Pi\left(S^{-1} R \otimes\right.$ $\bar{s})=0$. Hence if $f$ is injective, it must be true that $S^{-1} R \otimes \Pi \bar{s}=0$. Therefore, by the first part of the lemma, $S \cap(\cap(s)) \neq \varnothing$.

LEMMA 4.4. If $R$ is a commutative ring such that for every prime ideal $P,(R-P) \cap\left(\bigcap_{s \notin P}(s)\right) \neq \varnothing$, then every prime ideal is maximal. In particular, if $R$ is a domain then $R$ is a field.

Proof. If $t \in(R-P) \cap\left(\bigcap_{s \notin P}(s)\right)$ then $t+P \in \bigcap_{s \notin P}((s)+P) / P$. So $t+P \in\left(\left(t^{2}\right)+P\right) / P$. Hence there exists $r \in R$ such that $t-r t^{2}=$ $t(1-r t) \in P$. So $1-r t \in P$. This says $t+P$ is a unit in $R / P$.

If $s \in R-P$ then $t+P \in((s)+P) / P$. Since $t+P$ is a unit in $R / P$, $(s)+P=R$. Choose $b \in R$ such that $s b-1 \in P$. Then $(s+P)(b+P)=$ $1+P$, i.e., $s+P$ is a unit in $R / P$. Hence $P$ is maximal.

THeOREM 4.5. If $R$ is a commutative ring and $f$ is always injective then $R$ is artinian.

Proof. By Lemma $4.3(R-P) \cap\left(\bigcap_{s \notin P}(s)\right) \neq \varnothing$ for all prime ideals $P$. Therefore, every prime ideal is maximal (Lemma 4.4). But $R$ must be noetherian (Prop. 2.4). Since $R$ is noetherian with every prime ideal maximal, it is a standard result that $R$ must be artinian.

Since an artinian domain is necessarily a field we have the following immediate corollary.

COROLlary 4.6. If $R$ is a domain and $f$ is always injective then $R$ is a field.

As already remarked we do not know if the converse to Thm. 4.5 is true. There are, however, important classes of artinian rings over which $f$ is injective. For example, suppose $R$ is a proper quotient of a Dedekind domain. Then any $R$-module can be considered as a module, say $E$, over a Dedekind domain, this module having nontrivial annihilator. A classical result of Prüfer [10, Thm. 6] tells us that $E$ is a direct sum of cyclic submodules. Since $f$ is injective on each summand (Prop. 1.3), $f$ is injective on all of $E$ (Prop. 2.1).

A proper quotient of a Dedekind domain is a direct sum of rings each of whose lattices of ideals is finite and totally ordered by inclusion. 
Any ring, not necessarily commutative, whose left and right free modules of rank 1 have unique composition series has been called a serial ring [5].

THeOREM 4.7. (Eisenbud and Griffith, [5].) If $R$ is a serial ring then any right $R$-module is a direct sum of submodules with unique composition series.

In particular every module over a serial ring is a direct sum of finitely generated submodules. We have as an immediate consequence of this and Propositions 1.3 and 2.1, the following.

Proposition 4.8. If $R$ is a serial ring then $f$ is always injective.

\section{REFERENCES}

1. H. Bass, Finitistic homological dimension and a homological generalization of semiprimary rings, Trans. Amer. Math. Soc., 95 (1960), 466-488.

2. N. Bourbaki, Algèbre Commutative, Ch. 1 and 2, Hermann, Paris, 1961.

3. H. Cartan and S. Eilenberg, Homological Algebra, Princeton, 1956.

4. S. Chase, Direct products of modules, Trans. Amer. Math. Soc., 97 (1960), 457-473.

5. D. Eisenbud and P. Griffith, Serial rings, J. of Algebra, 17 (1971), 389-400.

6. E. Enochs, $A$ note on reflexive modules, Pacific J. Math., 14 (1964), 879-881.

7. L. Fuchs, Abelian Groups, Pergamon Press, New York, 1960.

8. - Infinite Abelian Groups, Vol. I, Academic Press, New York, 1970.

9. I. Kaplansky, Modules over Dedekind rings and valuation rings, Trans. Amer. Math. Soc., 72 (1952), 327-340.

10. - Infinite Abelian Groups, U. of Michigan Press, Ann Arbor, 1969.

11. D. Lazard, Sur les modules plats, C. R. Acad. Sc. Paris, t. 258 (29 Juin 1964), 6313-16.

12. E. Matlis, Injective modules over noetherian rings, Pacific J. Math., 8 (1958), 511-528.

13. R. Warfield, Jr., Purity and algebraic compactness for modules, Pacific J. Math., 28 (1969), 699-719.

Received August 21, 1972. This study is a doctoral dissertation prepared under the direction of Professor Edgar Enochs and submitted to the University of Kentucky. For the inspiration, guidance, and understanding by Professor Enochs throughout the development of this paper I am deeply grateful. 


\section{PACIFIC JOURNAL OF MATHEMATICS}

\section{EDITORS}

RICHARD ARENS (Managing Editor)

University of California

Los Angeles, California 90024

\section{R. A. Beaumont}

University of Washington

Seattle, Washington 98105

\section{J. DuGundJI*}

Department of Mathematics

University of Southern California

Los Angeles, California 90007

D. Gilbarg and J. Milgram

Stanford University

Stanford, California 94305

ASSOCIATE EDITORS
E. F. BECKENBACH
B. H. NeumanN
F. WOLF
K. YoSHIDA

\section{SUPPORTING INSTITUTIONS}

UNIVERSITY OF BRITISH COLUMBIA

CALIFORNIA INSTITUTE OF TECHNOLOGY

UNIVERSITY OF CALIFORNIA

MONTANA STATE UNIVERSITY

UNIVERSITY OF NEVADA

NEW MEXICO STATE UNIVERSITY

OREGON STATE UNIVERSITY

UNIVERSITY OF OREGON

OSAKA UNIVERSITY

\author{
UNIVERSITY OF SOUTHERN CALIFORNIA \\ STANFORD UNIVERSITY \\ UNIVERSITY OF TOKYO \\ UNIVERSITY OF UTAH \\ WASHINGTON STATE UNIVERSITY \\ UNIVERSITY OF WASHINGTON \\ AMERICAN MATHEMATICAL SOCIETY \\ NAVAL WEAPONS CENTER
}

The Supporting Institutions listed above contribute to the cost of publication of this Journal, but they are not owners or publishers and have no responsibility for its content or policies.

Mathematical papers intended for publication in the Pacific Journal of Mathematics should be in typed form or offset-reproduced, (not dittoed), double spaced with large margins. Underline Greek letters in red, German in green, and script in blue. The first paragraph or two must be capable of being used separately as a synopsis of the entire paper. Items of the bibliography should not be cited there unless absolutely necessary, in which case they must be identified by author and Journal, rather than by item number. Manuscripts, in duplicate if possible, may be sent to any one of the four editors. Please classify according to the scheme of Math. Rev. Index to Vol. 39. All other communications to the editors should be addressed to the managing editor, or Elaine Barth, University of California, Los Angeles, California, 90024.

50 reprints are provided free for each article; additional copies may be obtained at cost in multiples of 50 .

The Pacific Journal of Mathematics is issued monthly as of January 1966. Regular subscription rate: $\$ 48.00$ a year (6 Vols., 12 issues). Special rate: $\$ 24.00$ a year to individual members of supporting institutions.

Subscriptions, orders for back numbers, and changes of address should be sent to Pacific Journal of Mathematics, 103 Highland Boulevard, Berkeley, California, 94708.

\section{PUBLISHED BY PACIFIC JOURNAL OF MATHEMATICS, A NON-PROFIT CORPORATION}

Printed at Kokusai Bunken Insatsusha (International Academic Printing Co., Ltd.), 270, 3-chome Totsuka-cho, Shinjuku-ku, Tokyo 160, Japan.

* C. R. DePrima California Institute of Technology, Pasadena, CA 91109, will replace J. Dugundji until August 1974.

Copyright (C) 1973 by

Pacific Journal of Mathematics

All Rights Reserved 


\section{Pacific Journal of Mathematics}

\section{Vol. 49, No. $2 \quad$ June, 1973}

Wm. R. Allaway, On finding the distribution function for an orthogonal polynomial

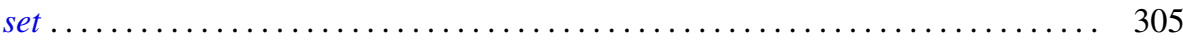

Eric Amar, Sur un théorème de Mooney relatif aux fonctions analytiques bornées... . 311

Robert Morgan Brooks, Analytic structure in the spectrum of a natural system . . . . 315

Bahattin Cengiz, On extremely regular function spaces . . . . . . . . . . . . . . 335

Kwang-nan Chow and Moses Glasner, Atoms on the Royden boundary . . . . . . . . . 339

Paul Frazier Duvall, Jr. and Jim Maxwell, Tame $Z^{2}$-actions on $E^{n} \ldots \ldots \ldots \ldots \ldots . .349$

Allen Roy Freedman, On the additivity theorem for $n$-dimensional asymptotic

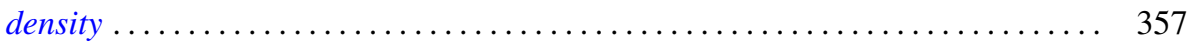

John Griffin and Kelly Denis McKennon, Multipliers and the group $L_{p}$-algebras . . . 365

Charles Lemuel Hagopian, Characterizations of $\lambda$ connected plane continua ....... 371

Jon Craig Helton, Bounds for products of interval functions . . . . . . . . . . . 377

Ikuko Kayashima, On relations between Nörlund and Riesz means . . . . . . . . . . 391

Everett Lee Lady, Slender rings and modules . . . . . . . . . . . . . . . . . . 397

Shozo Matsuura, On the Lu Qi-Keng conjecture and the Bergman representative

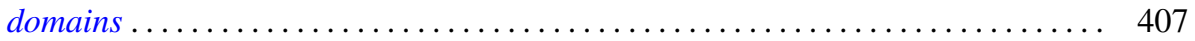

Stephen H. McCleary, The lattice-ordered group of automorphisms of an $\alpha$-set . . . 417

Stephen H. McCleary, o-2-transitive ordered permutation groups .......... 425

Stephen H. McCleary, o-primitive ordered permutation groups. II . . . . . . . . . 431

Richard Rochberg, Almost isometries of Banach spaces and moduli of planar

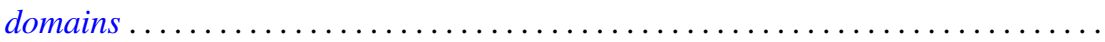

R. F. Rossa, Radical properties involving one-sided ideals . . . . . . . . . . . . . 467

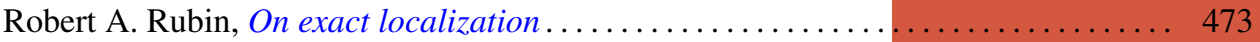

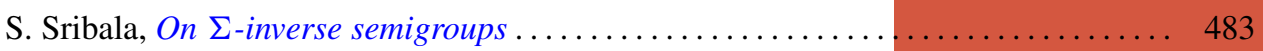

H. M. (Hari Mohan) Srivastava, On the Konhauser sets of biorthogonal polynomials suggested by the Laguerre polynomials ...................... 489

Stuart A. Steinberg, Rings of quotients of rings without nilpotent elements . ...... 493

Daniel Mullane Sunday, The self-equivalences of an $H$-space . . ............ 507

W. J. Thron and Richard Hawks Warren, On the lattice of proximities of $\check{C} e c h$

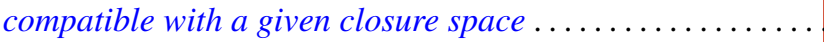

Frank Uhlig, The number of vectors jointly annihilated by two real quadratic forms determines the inertia of matrices in the associated pencil .

Frank Uhlig, On the maximal number of linearly independent real vectors annihilated simultaneously by two real quadratic forms ..............

Frank Uhlig, Definite and semidefinite matrices in a real symmetric matrix pencil . . 561

Arnold Lewis Villone, Self-adjoint extensions of symmetric differential operators . . . 569

Cary Webb, Tensor and direct products . ....................... 579

James Victor Whittaker, On normal subgroups of differentiable

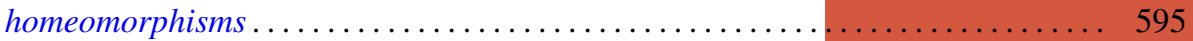

Jerome L. Paul, Addendum to: "Sequences of homeomorphisms which converge to

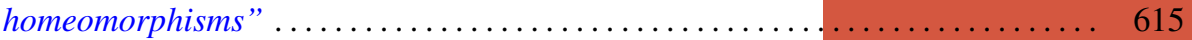

David E. Fields, Correction to: “Dimension theory in power series rings” ........ 616

Peter Michael Curran, Correction to: “Cohomology of finitely presented groups”. . . 617

Billy E. Rhoades, Correction to: “Commutants of some Hausdorff matrices” ...... 617

Charles W. Trigg, Corrections to: "Versum sequences in the binary system” ...... 619 